\title{
Various ways to build effective characteristics for a pipe made of a layered composite material
}

\author{
Tatiana Bobyleva ${ }^{1, *}$ and Alexey Shamaev ${ }^{2,3}$ \\ ${ }^{1}$ Moscow State University of Civil Engineering, Yaroslavskoe shosse, 26, Moscow, 129337, Russia \\ ${ }^{2}$ Ishlinsky Institute for Problems in Mechanics of the Russian Academy of Sciences, Moscow, \\ 119526, Russia \\ ${ }^{3}$ Lomonosov Moscow State University, Moscow, 119991, Russia
}

\begin{abstract}
Composite materials consisting of several phases are widely used in modern construction. Numerous experiments have shown that the properties of structurally heterogeneous materials can differ significantly from those of the individual components making up the composition. Besides, rapidly changing coefficients of differential equations describing such composite materials greatly complicate the solution of boundary value problems even with the help of computer calculation methods. Therefore, the homogenization method is used. In this paper the two approaches propose to obtain in explicit analytical form the effective model of the problem of loading a heterogeneous pipe made of layered material, provided that the elastic properties of the material depend only on the distance from the center of the section of the pipe. We point to a method that obviously leads to an analytical result. It follows from the article that it is possible to choose the function that determines the structure of the "winding" in such a way as to obtain the stiffness characteristics of the pipe as close as possible to the desired with fixed mass fractions of the materials used. A similar approach can be applied to the study of creep properties of pipes made of composite materials.
\end{abstract}

\section{Introduction}

Problems for products from heterogeneous materials, in particular pipes, arise in many areas of construction. To improve the strength characteristics of pipes during their manufacture, several layers of various materials are used, which together provide high tightness and strength. Layers often vary considerably in stiffness. Examples include fibrous composites formed by longitudinal-transverse winding. Such heterogeneity is the cause of their specific behavior during deformation. The method of asymptotic averaging turns such a material into a homogeneous one, which is described by averaged equations. This averaging method is stated, for example in $[1,2]$, one of the first applications of which was the problems of the theory of elasticity $[3,4]$. Several examples of its application are given in [5-8].

In this article we also want to consider the "composite" pipe from several materials. The load is applied in the form of the force field acting on the surface and distributed inside the volume. One of the tasks of this work is to replace a layered cylindrical pipe with a

\footnotetext{
* Corresponding author: tatyana2211@ outlook.com
} 
one-dimensional beam, which has corresponding effective characteristics for bending, tension, torsion in explicit analytical form (see subsection 2.2). A similar problem was considered in [9-13]. In this article, it was not a cylindrical tube, but a rod with an arbitrary periodic structure. According to the formulation of the problem, our task is a special case of [9]. In the case under consideration, we can explicitly construct the above-mentioned effective characteristics and solve the loading problem analytically. This is due to the fact that in this case it is possible to explicitly solve the auxiliary boundary value problems on a periodicity cell necessary for constructing effective characteristics. These boundary value problems have periodic boundary conditions along the elastic rod and the corresponding Neumann conditions on the lateral surface. In this case, by introducing cylindrical coordinates, we can reduce these auxiliary three-dimensional problems of the theory of elasticity to one-dimensional systems, where only the radial variable is an independent variable (the angular and axial variables are not included in this system of equations). And the systems of equations have a divergent form. As a result of such a structure, we can explicitly obtain an analytical solution. In this case, the condition of periodicity in the direction along the pipe is fulfilled automatically, since this solution does not depend on the axial coordinate. It is well known that the Neumann problem does not always have a solution. There are necessary and sufficient conditions for the existence of a solution connecting the boundary conditions with the right-hand side of the system. Auxiliary boundary value problems contain constant matrices. It is the choice of these constant matrices that will ensure that the solvability conditions are satisfied. The elements of these matrices will be the numbers that determine the effective characteristics of the heterogeneous pipe, namely, the effective indicators of flexural rigidity, tensile stiffness and torsion. We obtain these effective indicators and auxiliary functions in an explicit analytical form. Forces applied to the surface can be selected in various ways. It can simulate a force load applied in a direction perpendicular to the pipe and parallel to the pipe, as well as torsion around an axis directed along the pipe or perpendicular to it. The available analytical solutions will allow to evaluate qualitative and quantitative characteristics of obtained stress-strain states.

Another approach, considered in this paper and proposed as an "alternative", is to average the equations of elasticity theory for a cylindrical body with a rapidly oscillating periodic layered structure with the radial symmetry in a three-dimensional cylindrical region, without a transition to one-dimensional spatial variable structures. It is described in subsection 2.1 and is an implementation of the approach developed in [1] for analyzing layered structures. The result for further analysis requires the use of numerical methods using large finite elements or differential grids with a large step (much larger than the layer thickness). This drastically reduces the amount of computation compared to direct numerical calculation, but, of course, does not lead to an analytical representation for solutions. In this case, it is possible to obtain numerically a more complete picture for the stress-strain state of the cylindrical body under consideration, than this can be done in the transition to one-dimensional elastic models. The coefficients of the averaged system of elasticity theory will depend on the slow variable which is the distance to the axis of the cylinder.

\section{Problem specification and decision}

\subsection{The first approach}

Consider the system of equilibrium equations of the theory of elasticity [1] in Cartesian coordinates $x_{1}, x_{2}, x_{3}$ 


$$
\frac{\partial}{\partial x_{j}}\left(A^{i j}\left(\frac{\varphi(\vec{x})}{\varepsilon}\right) \frac{\partial \vec{u}}{\partial x_{i}}\right)=\vec{f}
$$

specified in a three-dimensional hollow cylinder (pipe) $P \equiv\left\{r_{0}<x_{1}^{2}+x_{2}^{2}<R^{2}, \quad x_{3} \in(0 ; z]\right\}$,

$(i, j=1,2,3)$. The dependence of the matrices $A^{i j}(t)$ on the scalar variable $t$ is assumed to be a periodic, $\varphi=\varphi\left(x_{1}, x_{2}\right)$ is the smooth function, vector $\vec{u}$ is the displacement vector, $\vec{f}$ is the vector of mass forces, $\varepsilon$ is the small parameter, $\varepsilon \in(0 ; 1)$. The case of a layered cylinder with a periodic layer structure corresponds to the function $\varphi=\sqrt{x_{1}^{2}+x_{2}^{2}}$. Further, we will denote as $\varphi_{\mathrm{k}}$ the derivative of the function $\varphi$ with respect to the variable $x_{k}(k=1$, $2,3)$, and also $y=\left(x_{1}, x_{2}, x_{3}\right)$.

We will define matrices

$$
\begin{gathered}
B_{\varepsilon}^{0}(t, y)=B^{0}\left(\frac{t}{\varepsilon}, y\right)=\left[\varphi_{l}(y) \varphi_{k}(y) A^{k l}\left(\frac{t}{\varepsilon}\right)\right]^{-1} \\
B_{\varepsilon}^{s}(t, y)=B^{s}\left(\frac{t}{\varepsilon}, y\right)=\left[\varphi_{l}(y) \varphi_{k}(y) A^{k l}\left(\frac{t}{\varepsilon}\right)\right]^{-1} \varphi_{p}(y) A^{p s}\left(\frac{t}{\varepsilon}\right), \\
=\varphi_{j}(y) A^{k l}\left(\frac{t}{\varepsilon}\right)\left[\varphi_{l}(y) \varphi_{k}(y) A^{k l}\left(\frac{t}{\varepsilon}\right)\right]^{-1} \varphi_{p}(y) A^{p s}\left(\frac{t}{\varepsilon}\right)-A^{i s}\left(\frac{t}{\varepsilon}\right) .
\end{gathered}
$$

In accordance with Theorem 7.13 [1], the set of matrices $\hat{A}^{i s}$ describing the "averaged" or "effective" properties of an elastic cylindrical body $P$ is given by the formulas

$$
\hat{A}^{i s}=\left\langle B^{i}\right\rangle^{*}\left\langle B^{0}\right\rangle^{-1}\left\langle B^{s}\right\rangle-\left\langle B^{i s}\right\rangle
$$

where $\left\langle B^{i}\right\rangle^{*}$ is the matrix conjugate to $\left\langle B^{i}\right\rangle$, and $\langle f\rangle$ means the period average of the function periodic in the variable $t:\langle f\rangle=\frac{1}{\tau} \int_{0}^{\tau} f(t) d t, \tau$ is the period value.

It is obvious that with a given periodic configuration of the layers and given elastic characteristics of each of the materials included in the "winding", all the coefficients of the matrices $\hat{A}^{i s}$ can be calculated explicitly. The corresponding stress-strain state of the cylinder can be calculated as a solution of the following system

$$
\frac{\partial}{\partial x_{i}}\left(\hat{A}^{i s} \frac{\partial \hat{u}}{\partial x_{s}}\right)=\vec{f}(\vec{x})
$$

with the corresponding boundary conditions at the ends and the lateral surface of the cylinder $P$. 
For our case of a circular section of a pipe, the coefficients of the matrices will only depend on the "slow" radial variable $r=\sqrt{x_{1}^{2}+x_{2}^{2}}$ and on $x_{1}, x_{2}$. This arises due to the dependence of matrix coefficients on the derivatives $\varphi_{\mathrm{k}}$ in the formulas $(2-4)$. The actual calculation of the stress-strain state of a "homogenized" cylinder can be done using, for example, by finite element method, which drastically reduces the amount of computation compared to the amount of computation needed to solve the original problem, which requires finite elements that are significantly smaller than width of each layer. In this case, we deal with calculations of the elastic deformed state of a three-dimensional body.

\subsection{The second approach}

Suppose that the thickness of an elastic cylindrical body (pipe) tends to zero, the layers do not have a periodic structure, but the "winding" has radial symmetry, that is, the coefficients of the matrices $A_{\varepsilon}^{i j}$ have the form $F\left(\frac{r}{\varepsilon}\right)$, where $r=\sqrt{x_{1}^{2}+x_{2}^{2}}, \varepsilon \rightarrow 0$.

Using the results of [9], we can approximately replace this "thin" elastic body with a onedimensional rod, the stress-strain state of which is described by functions of only one spatial variable $x_{3}$, and the "effective" equation is a rod (beam) equation that can be solved explicitly. To determine the characteristics of the Young's modulus and flexural rigidity, it is necessary, according to [9], to solve "auxiliary" problems for the functions $N_{1}, N_{2}, N_{3}, N_{4}$ :

$$
\begin{gathered}
\frac{\partial}{\partial \xi_{i}}\left(A^{i j}(\xi) \frac{\partial}{\partial \xi_{j}} N_{1}\right)=-\frac{\partial}{\partial \xi_{i}} A^{i 1}(\xi), \quad \xi \in Q, \\
\left.n_{k} A^{k j}(\xi) \frac{\partial}{\partial \xi_{j}} N_{1}\right|_{\Sigma}=-n_{k} A^{k 1}, \\
\frac{\partial}{\partial \xi_{i}}\left(\begin{array}{c}
\left.A^{i j}(\xi) \frac{\partial N_{2}}{\partial \xi_{j}}\right)=-\left(\frac{\partial}{\partial \xi_{i}}\left(A^{i 1}(\xi) N_{1}(\xi)\right)+A^{1 j}(\xi) \frac{\partial N_{1}}{\partial \xi_{i}}(\xi)+A^{11}(\xi)+h_{2}\right), \quad \xi \in Q \\
,\left.\quad n_{k} A^{k j}(\xi) \frac{\partial N_{2}}{\partial \xi_{j}}\right|_{\Sigma}=-n_{k} A^{k 1} N_{1}, \\
\frac{\partial}{\partial \xi_{i}}\left(A^{i j}(\xi) \frac{\partial N_{3}}{\partial \xi_{j}}\right)=-\left(\frac{\partial}{\partial \xi_{i}}\left(A^{i 1}(\xi) N_{2}(\xi)\right)+A^{1 j}(\xi) \frac{\partial N_{2}}{\partial \xi_{i}}(\xi)+A^{11} N_{1}+h_{3}\right), \quad \xi \in Q \\
\left.n_{k} A^{k j}(\xi) \frac{\partial N_{3}}{\partial \xi_{j}}\right|_{\Sigma}=-n_{k} A^{k 1} N_{2},
\end{array}\right.
\end{gathered}
$$


and

$$
\begin{aligned}
\frac{\partial}{\partial \xi_{i}}\left(A^{i j}(\xi) \frac{\partial N_{4}}{\partial \xi_{j}}\right)= & -\left(\frac{\partial}{\partial \xi_{i}}\left(A^{i 1}(\xi) N_{3}(\xi)\right)+A^{1 j}(\xi) \frac{\partial N_{3}}{\partial \xi_{i}}(\xi)+A^{11} N_{2}+h_{4}\right) \\
& \left.n_{k} A^{k j}(\xi) \frac{\partial N_{4}}{\partial \xi_{j}}\right|_{\Sigma}=-n_{k} A^{k 1} N_{3} .
\end{aligned}
$$

Here $B$ be a 1-periodic in the direction $x_{1}$ set in three-dimensional space, and domain $Q$ is $Q=\left\{\xi \in B: 0<\xi_{1}<1\right\}, \xi=\left(\xi_{1}, \xi_{2}, \xi_{3}\right)$, the symbol $\Sigma$ stands for the lateral boundary of $Q: \Sigma=\left\{\xi \in \partial B: 0<\xi_{1}<1\right\}$.

Let a layered tube has along its radius pairs of alternating homogeneous layers of two elastic isotropic materials (Fig.1). The problem in this case can be reduced to systems of ordinary differential equations by introducing a cylindrical coordinate system $(r ; \theta ; z)$ and solved explicitly.

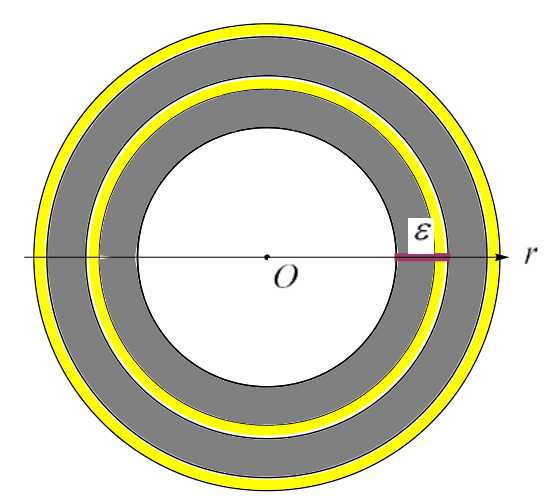

Fig. 1. The cross section of the layered pipe.

For such a problem Lame equations are [14]:

$$
\left\{\begin{array}{l}
(\lambda+2 \mu) \frac{\partial \Delta}{\partial r}-\frac{2 \mu}{r} \frac{\partial \omega_{z}}{\partial \theta}+2 \mu \frac{\partial \omega_{\theta}}{\partial z}=f_{r} \\
(\lambda+2 \mu) \frac{1}{r} \frac{\partial \Delta}{\partial \theta}-2 \mu \frac{\partial \omega_{r}}{\partial z}+2 \mu \frac{\partial \omega_{z}}{\partial r}=f_{\theta} \\
(\lambda+2 \mu) \frac{\partial \Delta}{\partial z}-\frac{2 \mu}{r} \frac{\partial\left(r \omega_{\theta}\right)}{\partial r}+\frac{2 \mu}{r} \frac{\partial \omega_{r}}{\partial \theta}=f_{z}
\end{array}\right.
$$


where $\vec{u}=\left(\begin{array}{l}u_{r} \\ u_{\theta} \\ u_{z}\end{array}\right)$ is the displacement vector, $\lambda, \mu$ are Lame parameters, $\vec{f}=\left(\begin{array}{c}f_{r} \\ f_{\theta} \\ f_{z}\end{array}\right)$ means the vector of mass forces. As is easy to see, components of the vector $\vec{f}$ depend only on the radial variable $r$. Then using the next formulas [9]

$$
\begin{aligned}
& \hat{c}_{1}=\frac{1}{|Q|} \int_{Q}\left[A^{1 j}(\xi)\left(\frac{\partial N_{1}}{\partial \xi}(\xi)+\delta_{1 i} E\right)\right]_{11} d \xi, \\
& H=\frac{1}{|Q|} \int_{Q}\left[A^{1 j}(\xi)\left(\frac{\partial N_{2}}{\partial \xi}(\xi)+\delta_{1 i} N_{1}(\xi)\right)\right]_{11} d \xi, \\
& \hat{c}_{2}=\frac{1}{|Q|_{Q}} \int_{Q}\left[A^{1 j}(\xi)\left(\frac{\partial N_{2}}{\partial \xi}(\xi)+\delta_{1 i} N_{2}(\xi)\right)\right]_{22} d \xi \\
& \hat{c}_{3}=\frac{1}{|Q|_{Q}} \int_{Q}\left[A^{1 j}(\xi)\left(\frac{\partial N_{3}}{\partial \xi}(\xi)+\delta_{1 i} N_{2}(\xi)\right)\right]_{33} d \xi,
\end{aligned}
$$

we can also explicitly obtain the elastic characteristics of an "effective" rod. In (8)-(11) the symbol $E$ stands for the unit matrix, and $|Q|$ stands for the volume of $Q$.

Then we can choose the function that determines the structure of the "winding" in order to get the stiffness characteristics as close as possible to the desired ones with fixed mass fractions of the materials used.

\section{Conclusion}

The approaches proposed in this paper propose to obtain in explicit analytical form the solution of the problem of loading a non-uniform pipe made of laminated material, provided that the elastic properties of the material depend only on the distance from the center of the section of the pipe. It is possible to obtain the elements of $h_{2}$ and $h_{3}$ matrices explicitly and they characterize the effective modulus of the pipe under tension, torsion, and bending. A similar approach can be applied in the future to study the creeping properties of a pipe made of composite materials. In fact, using the Laplace transform, we can reduce the problem of determining creeping deformations under various long-term pipe loads to the problem considered in this article. In this case, after the Laplace transform, we obtain the problem of elastic equilibrium with coefficients depending on the complex parameter. The solution of such a task will in fact be no different from what is proposed in this work. Then, it will be necessary to perform the inverse Laplace transform, which is possible, for example, when the initial creep kernels (relaxation) are sums of decreasing exponents. Inverse Laplace transform for such exponential kernels can be made explicit.

\section{References}

1. O. A. Oleynik, G.A. Iosif'yan and A.S. Shamaev, Mathematical problems in elasticity and homogenization (Elsevier, North-Holland, 1992) 
2. D. I. Bardzokas and A. I. Zobnin, Mathematical modeling of physical processes in composite materials of periodic structure (URSS, Moscow, 2005)

3. B. E. Pobedrya, Mechanics of composite materials (MSU, Moscow ,1984)

4. R. M. Christensen, Mechanics of composite materials (Dover, New York, 2005)

5. T. N. Bobyleva, MATEC Web of Conf. 86, 01024 (2016)

6. T. N. Bobyleva and A. S. Shamaev, MATEC Web of Conf. 251, 04039 (2018)

7. T. N. Bobyleva and A. S. Shamaev, Soil Mechanics and Foundation Engineering 54, 224 (2017)

8. T. N. Bobyleva and A. S. Shamaev, IFAC-PapersOnLine 51, 138 (2018)

9. D. Lukkassen, A. Meidell, A. L. Piatnitski and A. S. Shamaev, Applicable Analysis 88, 15637 (2009)

10. D. Lukkassen, A. Meidell, A. L. Piatnitski and A. S. Shamaev, Mathematical Models and Methods in Applied Sciences 19 (4), 501 (2009)

11. G. P. Panasenko, Russian Journal of Math. Physics 2, 325 (1994)

12. G. P. Panasenko, Russian Journal of Math. Physics 4, 87 (1996)

13. M. V. Kozlova and G. P. Panasenko, Comput. Math. Math. Phys. 31, 128 (1992)

14. A. E. H. Love, Treatise on the mathematical theory of elasticity (Dover Publ., New York, 1944) 